\title{
Entropic attraction of adhesion bonds toward cell boundaries
}

\author{
Noam Weil and Oded Farago ${ }^{1}$ \\ ${ }^{1}$ Department of Biomedical Engineering, Ben Gurion University, Be'er Sheva 84105, Israel
}

\begin{abstract}
Adhesion bonds between membranes and surfaces are attracted to each other via effective interactions whose origin the entropy loss due to the reduction in the amplitude of the membrane thermal fluctuations in the vicinity of the adhesion bonds. These fluctuation-induced interactions are also expected to drive the adhesion bonds toward the rim of the cell, as well as toward the surfaces of membrane inclusions. In this paper, we analyze the attraction of adhesion bonds to the cell inner and outer boundaries. Our analysis shows that the probability distribution function of a single (diffusing) adhesion bond decay algebraically with the distance from the boundaries. Upon increasing the concentration of the adhesion bonds, the attraction to the boundaries becomes strongly self-screened.

PACS numbers:
\end{abstract}

\section{INTRODUCTION}

Biological membranes are essential to every living organism. They consist of a bilayer of amphiphilic lipids, proteins and cholesterol, and serve as a barrier between the cytosol and the extracellular matrix in biological cells [1]. In eukaryotic cells, membranes surround the organelles, allowing for organization of biological processes through compartmentalization. Membranes are involved in a variety of cellular processes, including the transition of nutrients in and out of the cell, signal transduction and communication 2].

One of the processes taking place at the cell membrane is cell adhesion. This process, during which the cell membrane is attracted to another interface (which may be the membrane of another cell) can, in principle, be facilitated by non-specific attractive interactions (e.g., Coulomb and van der Waals interactions). Most often, however, cell adhesion is mediated by specific binding between receptors that reside on the membrane surface and ligands located on the opposite surface [3]. Specific adhesion usually occurs in regions with high density of receptors and ligands. When facing a surface with enough ligands, the receptors may cluster into highly concentrated adhesion domains to establish strong binding [4, 5]. Specific bioadhesion occurs in a variety of cellular processes, including binding of white blood cells to pathogens [6], binding and fusion of drug carrier liposome to target cells [7], cadherin-mediated adhesion of neighboring cells [8], focal adhesion of cells to the extracellular matrix [9], and cell signaling.

Adhesion induced domain formation results from both the activity of the actin cytoskeleton, and from attractive interactions which are mediated by the membrane curvature elasticity [10]. The membrane-mediated interactions give rise to two distinct, but not entirely unrelated, mechanisms facilitating cluster formation. The first mechanism is the Casimir-like attraction between two bonds, which originates from the reduction in the amplitude of the membrane thermal fluctuations in the vicinity of the bonds and the associated entropy loss. The resulting increase in the free energy of the membrane is minimized when the two bonds come within close proximity of each other, in which case the area where the thermal fluctuations are suppressed is reduced [11]. The second mechanism is related to the tendency of new bonds to form next to existing ones. This "binding cooperativity" effect stems from the fact that the ligand-receptor binding probability is highest when the separation between the ligand and the receptor on the opposite surfaces is close to the bond length - conditions which are likely to occur near already existing bonds [12]. In addition, since the formation of new bonds leads to an overall reduction in the membrane roughness, it also increases the binding probability and assists the formation of even more bonds 13. Generally speaking, the first mechanism is likely to contribute to the aggregation of irreversible bonds with large binding energies, while the second one is probably more relevant to the formation of metastable domains of weaker reversible bonds.

In a previous publication we proposed a framework for studying the Casimir-like interactions between adhesion bonds 14]. Our analysis is based on the following idea: The Casimir-like interactions are induced by the membrane thermal fluctuations whose amplitude is suppressed due to the presence of adhesion bonds. To calculate the free energy cost of a given distribution of adhesion bonds, a free energy density is assigned to each unit area of the fluctuating membrane, and the free energy density is integrated over the entire membrane area. The free energy density reflects the extend by which the amplitude of the fluctuations is locally reduced compared to the fluctuations of a free (non-adhering) membrane. The key point of the method is the assumption amplitude of thermal fluctuations depends on the distance $d_{\min }$ to the nearest adhesion bond. Accordingly, the free energy density associated with each unit area of the membrane is given by (see derivation in [14]):

$$
V(r)=\left(\frac{k_{B} T}{\pi}\right)\left(\frac{1}{d_{\min }}\right)^{2}
$$


The total attachment free energy is given by

$$
F=\int V\left(d_{\min }\left(\overrightarrow{r^{\prime}}\right)\right) d \overrightarrow{r^{\prime}}=\sum_{i} \frac{k_{B} T}{\pi}\left(\frac{l}{d_{\min }}\right)^{2}\left(1-s_{i}\right),
$$

where the integration is carried over the entire membrane area accept for the immediate vicinities of the adhesion bonds. The sum on the r.h.s. of eq. 2 is the discrete ana$\log$ of the integral, which is useful for lattice simulations and numerical integrations. In the sum, the variable $s_{i}$ takes the value $s_{i}=1$ for the sites occupied by adhesion bonds and $s_{i}=0$ for an empty sites that represent fluctuating unit areas of size $l^{2}$. Only the latter contribute to the sum.

We studied the model defined by eq. 2 by using both a mean field analysis and Monte Carlo simulations 14]. Our analysis revealed (in agreement with other theoretical studies [15, 16]) that the fluctuation-induced interactions are not strong enough to allow the formation of adhesion domains. However, usually the adhesion bonds also interact with each other via short range attractive hydrophobic and depletion forces [17]; and when these residual interactions are introduced into the model, condensation of adhesion domains becomes thermodynamically possible [14, 15]. Our analysis showed that the fluctuation-induced Casimir-like interactions greatly reduce the strength of the residual attractive interactions required for cluster formation by almost half an order of magnitude, to below the thermal energy $k_{B} T$.

In this work we take a step forward and study the formation of adhesion domains in cellular membranes which also include trans-membrane proteins. Since these inclusions significantly influence the membrane spectrum of thermal fluctuations [18], their presence is expected to modify the fluctuation-induced interaction between adhesion bonds and their aggregation behavior. In this context, it is worthwhile to mention that there is a great bulk of experimental [19 21] and theoretical 222 25] works on the fluctuation-mediated potential between the inclusions themselves. Here, we tackle a different problem and explore how a single inclusion affects the aggregation of adhesion bonds. Since the formation of adhesion cluster is driven by the tendency to localize the reduction of the thermal fluctuations in a restricted area, it is natural to expect that adhesion bonds would exhibit affinity to the surface of the inclusion. Physically, the effect of an inclusion on the thermal fluctuations should be similar to that of a cluster of adhesion bonds of the same size. However, as discussed above, without direct residual interactions the system is in the gas-phase and a cluster of adhesion bonds is not stable. Therefore, the presence of the inclusion is not equivalent to the formation of a cluster, which is only transient.

The paper is organized as follows: In sec. III, we examine the attraction between a circular inclusion and a single adhesion bond. We derive the probability distribution of the adhesion bond from the center of the inclusion. We show that in contrast to the weak attraction between two

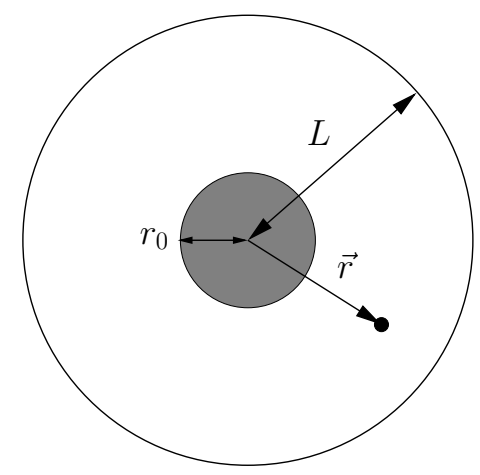

FIG. 1: Schematic of the system under investigation, consisting of a circular membrane of radius $L$ with a circular inclusion of radius $r_{0}$ at the center. The membrane is supported by a flat surface to which it is attached by a single adhesion bond located at $\vec{r}$.

adhesion bonds (see ref. [26]), the fluctuation-induced attraction between the adhesion bond and a large inclusion is sufficiently strong to keep the adhesion bond in the vicinity of the inclusion. In sec. [II] we study the distribution and aggregation of many adhesion bonds in the presence of the inclusion. Using Monte Carlo simulations with different boundary conditions and different adhesion bonds densities, we demonstrate that the strong attractive potential calculated in sec. III is screened and felt only by the adhesion bonds located near the surface of the inclusion. In sec. IV] we summarize and discuss our results.

\section{THE BOND-INCLUSION PAIR INTERACTION}

We consider the model system shown schematically in fig. 1. consisting of a circular membrane of radius $L$, a circular inclusion of radius $r_{0} \ll L$ fixed at the center of the membrane, and a single adhesion bond located at $\vec{r}$. The total attachment free energy of the system is given by eq. 2

$$
F\left(\vec{r}, r_{0}, L\right)=\int_{r_{0}+l}^{L-l} \frac{k_{B} T}{\pi d_{\min }^{2}\left(\overrightarrow{r^{\prime}}, \vec{r}, r_{0}, L\right)} d \overrightarrow{r^{\prime}},
$$

where the integration is carried over the whole area of the membrane, excluding regions of microscopic size $l$ near the inner $\left(\left|\overrightarrow{r^{\prime}}\right|=r_{0}\right)$ and outer $\left(\left|\overrightarrow{r^{\prime}}\right|=L\right)$ boundaries, and in the vicinity of the adhesion bond $\left(\overrightarrow{r^{\prime}}=\vec{r}\right)$. In eq. 3. $d_{\text {min }}$ denotes the distance of a given point $\overrightarrow{r^{\prime}}$ on the membrane to the nearest "obstacle", which may be either the adhesion bond or the surface of the inclusion. The assumption underlying eq. 3 is that the membrane separation at the inclusion is the same as the length of the adhesion bond. This feature is also likely to contribute to the binding cooperativity mechanism mentioned above in section [. which we do not consider in this 

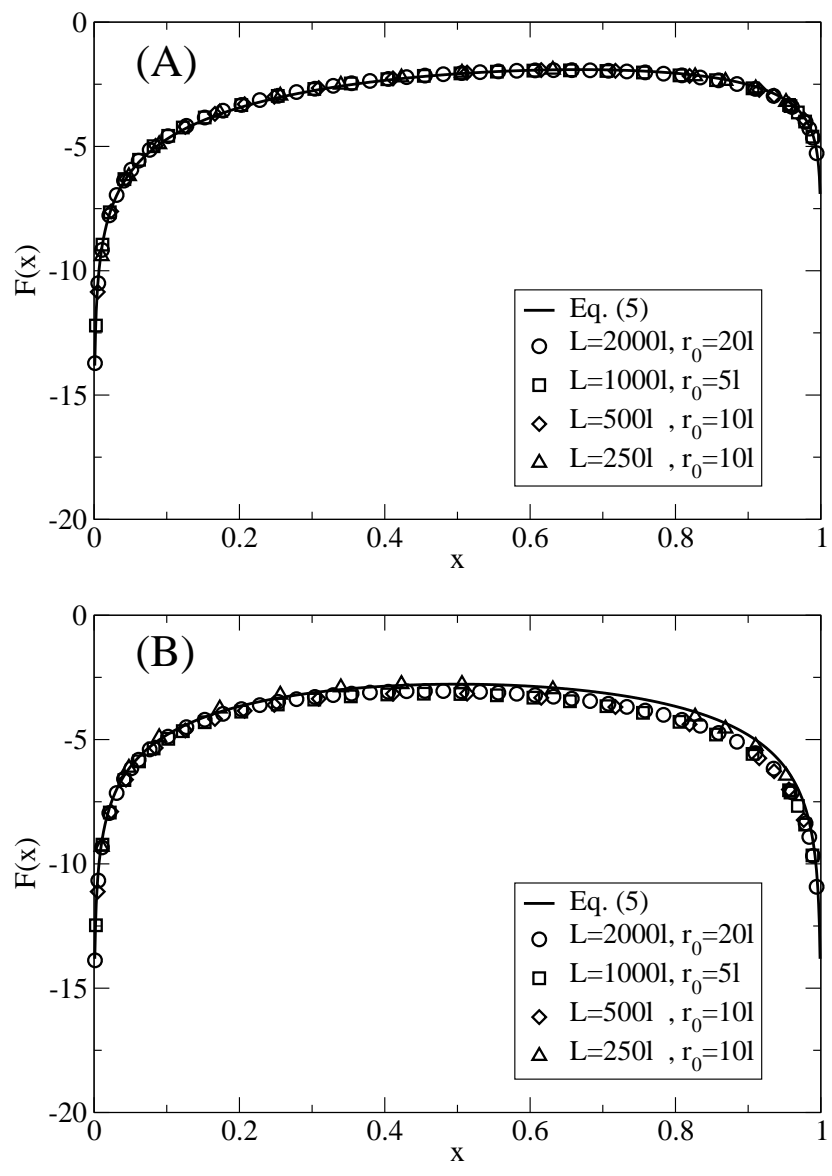

FIG. 2: Data collapse of the attachment free energy for different values of $|\vec{r}|, r_{0}$, and $L$, as a function of the dimensionless distance $x=\left(|\vec{r}|-r_{0}\right) /\left(L-r_{0}\right)$. The data in (A) and (B) corresponds to systems with open and closed outer boundaries, respectively.

work. Had there been a length mismatch between the inclusion and the adhesion bonds, it would have involved a bending elasticity energy cost that would weaken the fluctuation-induced attraction. In a binary mixtures of adhesion bonds, the inclusion is likely to have a higher affinity to the adhesion bonds with the smaller length mismatch, which may lead to phase segregation in the mixture [27, 28].

In what follows, we consider membranes with both "open" and "closed" outer boundaries. At a closed boundary, the membrane is attached to a frame, whereas at an open boundary, the membrane fluctuates freely. Thus, the former is also considered as an obstacle, while the latter is not. The open and closed boundary conditions represent two limiting cases. Cellular membranes are attached to the actin cytoskeleton located mainly at the periphery of the cell., which corresponds to an intermediate case between open and closed boundary conditions.

The integral in eq. 3 has been evaluated numerically for different values of $r_{0}$ and $L$, and for $r_{0}<|\vec{r}|<L$. Our calculations reveal that the attachment free energy

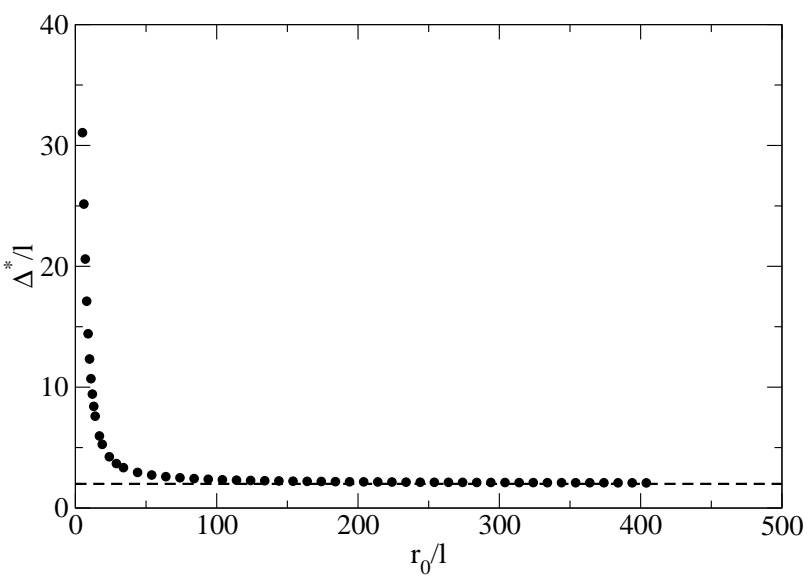

FIG. 3: The width $\Delta^{*}$ of the shell around the inclusion, as a function of the radius of the inclusion $r_{0}$. The dashed horizontal line corresponds to the solution of eq. 9. $\Delta^{*}=2 l$, for asymptotically large $r_{0}$.

is very well approximated by the following expression

$$
\begin{aligned}
F\left(\vec{r}, r_{0}, L\right) & \sim 2 k_{B} T \ln \frac{\left(|\vec{r}|-r_{0}\right)}{l} \\
& +n k_{B} T \ln \frac{(L-|\vec{r}|)}{l}+C\left(r_{o}, L\right),
\end{aligned}
$$

with $n=1$ for an open outer boundary, and $n=2$ for a closed boundary. Introducing the dimensionless distance $0<x=\left(|\vec{r}|-r_{0}\right) /\left(L-r_{0}\right)<1$, eq. 4 can be also expressed as

$$
\begin{aligned}
F(x) & \equiv F\left(\vec{r}, r_{0}, L\right)-\ln \left(\frac{L-r_{0}}{l}\right)^{2+n}-C\left(r_{o}, L\right) \\
& \sim 2 k_{B} T \ln (x)+n k_{B} T \ln (1-x) .
\end{aligned}
$$

Our numerical results along with eq. 5] are plotted in figs. 2(A) and 2(B) for an open and closed boundary, respectively. When the adhesion bond is located far away from the outer boundary, i.e. for $x \ll 1$, the attachment free energy is dominated by the first term $F \simeq 2 k_{B} T \ln (x)$, which has been previously derived as the pair potential of mean force between two adhesion bonds [26]. In the vicinity of an outer closed boundary $(x \lesssim 1)$, one gets $F \simeq 2 k_{B} T \ln (1-x)$, which implies that the adhesion bond is attracted to the nearest point on the outer closed boundary as if this point is another adhesion bond. For an open boundary, the prefactor of the second term in eq. 5 is reduced by half from $n=2$ to $n=1$. This can be rationalized by noticing that an open boundary is identical to the midplane between two adhesion bonds in a twice larger system. The pair potential between the adhesion bond and its image is $2 k_{B} T \ln [2(1-x)]=2 k_{B} T \ln [(1-x)]+C$, and this energy is equally divided between the real and imaginary halves of the system.

How strong is the attraction between the adhesion bond and the inner inclusion? From eq. 4 we 
find that the probability distribution function $P(\vec{r}) \sim$ $\exp \left[-F\left(\vec{r}, r_{0}, L\right) / k_{B} T\right]$ of the adhesion bond is given by

$$
P(\vec{r})=\frac{1}{Z} \frac{1}{\left(|\vec{r}|-r_{0}\right)^{2}(L-|\vec{r}|)^{n}},
$$

where the normalization factor is

$$
Z=\int_{r_{0}+l}^{L-l} \frac{2 \pi r d r}{\left(r-r_{0}\right)^{2}(L-r)^{n}}
$$

In the case of an open outer boundary $(n=1)$, the mean distance between the adhesion bond and the surface of the inner inclusion is given by

$$
\langle r\rangle-r_{0} \equiv \Delta=\frac{\left(L^{2}-r_{0}^{2}\right) \ln \left(\frac{L-r_{0}-l}{l}\right)}{2 L \ln \left(\frac{L-r_{0}-l}{l}\right)+r_{0}\left(r_{0}-L\right)\left[\frac{2 l+r_{0}-L}{l\left(L-r_{0}-l\right)}\right]},
$$

which for $L \gg r \equiv|\vec{r}| \gg r_{0}$, simplifies to $\Delta \sim L / 2$. The fact that the mean separation $\Delta$ grows linearly with the size of the membrane may be considered as an indication that the adhesion bond is not bound to the inclusion. However, a closer inspection of eq. 6 reveals that the adhesion bond spends most of its time near the boundary of the inner inclusion. To quantify this phenomenon, we introduce the length scale $\Delta^{*}$, which is the width of the shell around the inner inclusion where the probability to find the adhesion bond is 0.5 . The length $\Delta^{*}$ is determined by solving the equation

$$
P_{\mathrm{acc}}\left(\Delta^{*}\right)=\int_{r_{0}+l}^{r_{0}+\Delta^{*}} 2 \pi|\vec{r}| P(|\vec{r}|) d r=\frac{2 L \ln \left(\frac{\Delta^{*}}{l}\right)+r_{0}\left(r_{0}-L\right)\left(\frac{l-\Delta^{*}}{l \Delta^{*}}\right)}{2 L \ln \left(\frac{L-r_{0}-l}{l}\right)+r_{0}\left(r_{0}-L\right)\left[\frac{2 l+r_{0}-L}{l\left(L-r_{0}-l\right)}\right]}=0.5
$$

Our results for $\Delta^{*}$ are plotted in fig. 3 as a function of $r_{0}$ and for $L \gg r_{0}$. The results shows that $\Delta^{*}$ is a monotonically decreasing function of $r_{0}$. For very small inclusions of size $r_{0} \sim l$, the length $\Delta^{*} \gg l$. This result is consistent with the observation of ref. [26] that the fluctuationmediated pair potential is too weak to bind two adhesion bonds to each other. For inclusions of size $r_{0} \gtrsim 20 l$, the length $\Delta^{*}<5 l$; and for asymptotically large inclusions $\left(r_{0} \rightarrow \infty\right), \Delta^{*} \rightarrow 2 l$. In other words, although $\Delta \sim L / 2$, the adhesion bond is likely to be found within a thin shell around the surface of a sufficiently large inclusion.

For a closed outer boundary, the radial distribution function of the adhesion bond

$$
g(r) \equiv 2 \pi r P(|\vec{r}|)=\frac{r}{\left(r-r_{0}\right)^{2}(L-r)^{2}}\left\{\frac{\left(L-r_{0}\right)^{3}}{\left(L+r_{0}\right)\left[2 \ln \left(\frac{L-r_{0}-l}{l}\right)+\left(r_{0}-L\right)\left(\frac{2 l+r_{0}-L}{l\left(L-r_{0}-l\right)}\right)\right]}\right\} .
$$

(The expression appearing in braces in eq. 10 is a normalization constant that depends on $r_{0}$ and $L$ ). The radial distribution function $g(r)$, which is plotted in fig. 4 for $r_{0}=4 l$ and $L=100 L$, increases rapidly as one approaches both the inner and outer boundaries of the membrane. However, unlike the probability density per unit area $P(\vec{r})$ which is symmetric with respect to the mid-radius $r_{m}=\left(L+r_{0}\right) / 2$, the radial distribution function $g(r) \sim r P(|\vec{r}|)$ is much higher near the outer boundary than the inner one. This, of course, is directly related to the fact that there is simply more membrane area near the outer boundary. In the example plotted in fig. $4\left(r_{0}=4 l, L=100 l\right)$, the probabilities to find the ad- hesion bond within a shell of size $4 l$ around the outer and inner boundaries are $72.5 \%$ and $4.5 \%$, respectively. For a bigger membrane of size $L=1000 l$ (which, assuming that $l$ is of the order of a few nanometers, is a reasonable estimate for the size of the cell plasma membrane), these probabilities change to $79 \%$ and $0.5 \%$, respectively. These numbers suggest that the adhesion bond is likely to be "adsorbed" at the outer boundary. This, however, is only part of the story. If the adhesion bond is located near the inner boundary, it is not going to easily escape to the outer rim. The inset on fig. 4 shows the potential of mean force $F_{g}(r) \equiv-k_{B} T \ln [g(r)]$, exhibiting a free energy barrier of $\sim 4 k_{B} T$ that blocks the migra- 


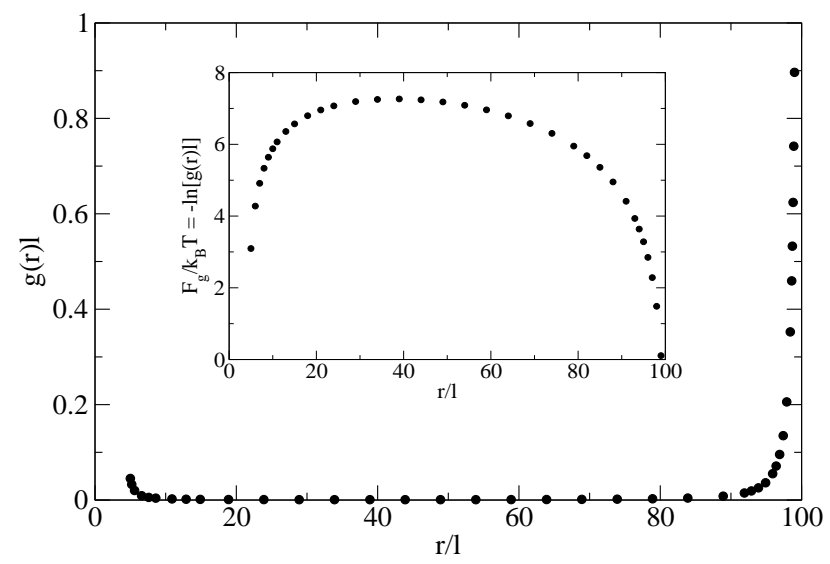

FIG. 4: The radial distribution function $g$ as a function of $r$, for $r_{0}=4 l$ and $L=100 l$. The inset shows the corresponding free energy, $F_{g}=-k_{B} T \ln [g(r) l]$.

tion of the adhesion bond from the inner boundary to the outer one. The barrier on the opposite direction is of $\sim 7 k_{B} T$. For $L=1000 l$, the free energy barriers increase to $\sim 7 k_{B} T$ and $\sim 12 k_{B} T$ for the escape from the inner and outer boundaries, respectively. Thus, as in the case with an open boundary discussed above, an adhesion bond located near the inner boundary may remain trapped there for relatively long times.

\section{DISTRIBUTION OF ADHESION BONDS AROUND AN INCLUSION}

The previous section dealt with the localization of a single adhesion bond near the boundaries of the system. Cellular adhesion, however, involves an ensemble of adhesion bonds which may cluster into adhesion domains. In a previous work [14], we demonstrated that the formation of adhesion domains cannot be induced by the Casimir-like interactions alone, and that it requires the existence of residual attractive interactions between the adhesion bonds. Here, we wish to understand whether this picture is changed by the presence of a membrane inclusion that serve as a potential nucleation seed for an adhesion domain. One can envision a scenario where a thin shell of adhesion bonds is formed around the inclusion, effectively increasing its size and promoting the recruitment of more adhesion bonds. The opposite scenario, in which the addition of adhesion bonds self-screen the attraction toward the inclusion, is also plausible. In order to investigate this issue, we performed Monte Carlo lattice simulations of the model defined by eq. 2 on circular systems with $r_{0}=4 l$ and $L=100 l$ (where $l$ is the lattice spacing). Our simulations were conducted at relatively low concentrations of adhesion bonds, $\phi=0.01$ and $\phi=0.05$, with both open and closed boundary conditions.

We measured the number density (per unit area) of adhesion bonds as a function of $\vec{r}$. To allow compar-
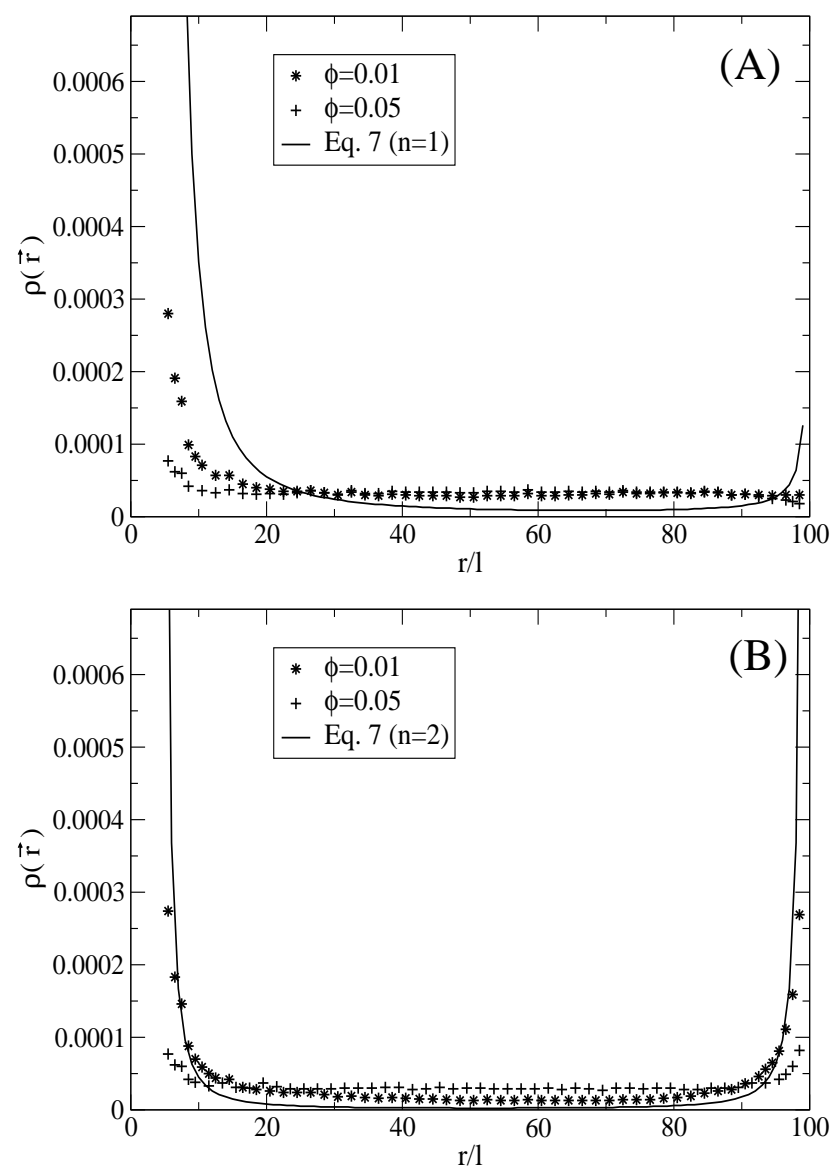

FIG. 5: The normalized number density $\rho(\vec{r})$ as a function of $r=|\vec{r}|$. Data shown in the figure corresponds to $r_{0}=4 l$ and $L=100 l$, for systems with (A) open and (B) closed outer boundaries. The solid lines depict the probability distribution of a single adhesion bond.

ison with the probability distribution $P(\vec{r})$ (eq. 6), we normalized the number density to the total number of adhesion bonds. Our results for the normalized number density, $\rho(\vec{r})$, are depicted in fig. 5(A) and 5(B) for open and closed boundaries, respectively. Both figures demonstrate that the fluctuation-induced attraction to the boundaries is self-screened by the presence of adhesion bonds. Comparison of our results for $\phi=0.01$ (depicted by stars in both figures) and $\phi=0.05$ (pluses), with eq. 6 for a single adhesion bond (solid lines), reveals that the self-screening effect is increased with the increase in the concentration $\phi$. For an open boundary, the slight increase in $P(\vec{r})$ near the outer boundary disappears already at $\phi=0.01$. For $\phi=0.05$, the density $\rho(\vec{r})$ is almost constant with no significant affinity of adhesion bonds toward the inner boundary. The same trends are also observed in the case of a closed boundary, where $P(\vec{r})$ increases very sharply near both boundaries. When the concentration $\phi$ increases, these maxima are quickly lowered, and the number density $\rho(\vec{r})$ becomes almost uniform. 


\section{SUMMARY AND DISCUSSION}

In this work we continued our investigation of the entropic, fluctuation-induced, attraction between adhesion bonds in supported biological membranes. The focus here was on the possible role played by the membrane boundaries as nucleation seeds for the formation of adhesion domains. Residence of adhesion bonds near the cell boundaries is thermodynamically favorable because it lowers the entropic cost associated with the suppression of the membrane thermal fluctuations around the adhesion bonds. We started our investigation by looking at the pair interaction between an adhesion bond and a circular membrane inclusion, and calculating the distribution function of the adhesion bond around the inclusion. In the case of an open outer cell boundary, the probability density of the adhesion bond decays algebraically with the distance from the surface of the inclusion. Although the mean pair distance grows linearly with the size of the membrane, the distribution function is such that the adhesion bond spends most of its time in the immediate vicinity of the inclusion. When the outer membrane boundary is closed, the probability distribution per unit area is symmetric with respect to the mid radius. In this case, the adhesion bond is likely to be found near the outer boundary, which is much larger than the inner one. Diffusion of the adhesion bonds between the two boundaries is limited by the existence of a substantial free energy barrier in the middle of the membrane.
Does this analysis imply that the cell boundaries can serve as nucleation seeds for adhesion domains? Perhaps yes, but probably not due to the fluctuation-induced mechanism alone. Our simulation results show that even at small densities of adhesion bonds, the entropic attraction toward the cell boundaries are entirely self-screened. This observation is consistent with our previous study of systems with periodic boundary conditions (no boundaries), where it has been found that the fluctuationinduced interactions are not sufficient to allow the formation of adhesion domains. In other words, without additional attractive interactions, the problem of aggregation of adhesion bonds is similar to the problem of gas to liquid condensation above the transition temperature. Introducing a nucleation seed into such a system without changing the temperature is not going to drive the system into the condensed phase. We leave for a future work the problem whether domain formation occurs at high density of inclusions whose inter-distances are smaller than the screening length for the adhesion bonds. Another aspect of the problem that needs to be explored is whether the existence of direct residual attractive interaction between the adhesion bonds and the inclusion can facilitate cluster formation, even in the absence of direct attractive interactions between the adhesion bonds themselves.

We thank Haim Diamant and Thomas Weikl for helpful comments. This work was supported by the Israel Science foundation (Grant Number 946/08).
[1] B. Alberts, D. Bray, J. Lewis, M. Raff, K. Roberts, and J. D. Watson, Molecular Biology of the Cell (Garland, New York, 1994).

[2] M. C. Beckerle (Ed.), Cell Adhesion (Oxford University Press, Oxford, 2001).

[3] D. A. Lauffenburger and J. Linderman, Receptors: Models for binding, Trafficking, and Signaling (Oxford University Press, Oxford, 1995).

[4] A.-S. Smith and U. Seifert, Soft Matter 3, 275 (2007).

[5] T. R. Weikl, M. Asfaw, H. Krobath, B. Röżycki and R. Lipowsky, Soft Matter 5, 3213 (2009).

[6] V. Naggli, Int. J. Biochem. Biochem Cell Biol. 35, 1619 (2003).

[7] T. M. Allen and P. R. Cullis, Science 303, 1818 (2004).

[8] K. Giehl and A. Menke, Front. Biosci. 13, 3975 (2008).

[9] B. Geiger, A. Bershadsky, R. Pankov, and K. M. Yamada, Nat. Rev. Mol. Cell Biol. 2, 793 (2001).

[10] H. Delanoë-Ayari, A. Al Kurdi, M. Vallade, D. GulinoDebrac, and D. Riveline, Proc. Nat. Acad. Sci. USA 101, 2229 (2004).

[11] R. Bruinsma, M. Goulian, and P. Pincus, Biophys. J 67, 746 (1994).

[12] E. Reister, T. Bihr, U. Seifert, and A. S. Smith, New J. Hpys. 13, 025003 (2011).

[13] H. Krobath, B. Różycki, R. Lipowsky, and T. R. Weikl, Soft Matter 5, 3354 (2009).

[14] N. Weil and O. Farago, Eur. Phys. J. E 33 (1), 81 (2010).

[15] T. R. Weikl and R. Lipowsky, in: A. L. Liu (Ed.) $A d-$ vances in Planar Lipid Bilayers and Liposomes, Vol. 5 (Elsevier, Amsterdam, 2007).

[16] T. Speck, Phys. Rev. E 83, 050901(R) (2011).

[17] J. Israelachvili, Intermolecular and Surface Forces (Academic Press, London, 1985).

[18] C. D. Santangelo and O. Farago, J. Computer-Aided Mater. Des. 14, 103 (2007).

[19] J. Salafsky, J. T. Groves, and S. G. Boxer, Biochemistry 35, (1996) 14773 (1996).

[20] R. Parthasarathy and J. T. Groves, J. Phys. Chem. B 110 (16), 8513 (2006).

[21] N. Taulier, M. Waks, T. Gulik-Krzywicki, and W. Urbach, Europhys. Lett. 59 (1), 142 (2002).

[22] R. Bruinsma and P. Pincus, Curr. Opin. Solid State Mater. Sci. 1,401 (1996).

[23] J.-M. Park and T. C. Lubensky, J. Phys. I 6, 1217 (1996).

[24] M. Kardar and R. Golestanian, Rev. Mod. Phys. 71, 1233 (1999).

[25] K. S. Kim, J. Neu, and G. Oster, Phys. Rev. E 61(4), 4281 (2000).

[26] O. Farago, Phys. Rev. E 81, 050902(R) (2010).

[27] B. Różycki, R. Lipowsky, and T. R. Weikl, New J. Phys. 12, 095003 (2010)

[28] N. J. Burroughs1, K. Köhler, V. Miloserdov, M. L. Dustin, P. A. van der Merwe, and D. M. Davis, Plos. Comut. Biol. 7, e1002076 (2011). 